\title{
Cáncer del tracto digestivo: asociación entre el estado nutricional y la capacidad funcional
}

\author{
Elizabeth Pérez-Cruz y Christian Patricio Camacho-Limas \\ Unidad de Soporte Nutricional y Metabolismo, Hospital Juárez de México, Ciudad de México, México
}

\section{Resumen}

Objetivo: Determinar el estado nutricional y su asociación con la capacidad funcional en pacientes con cáncer del tracto digestivo. Métodos: Estudio retrospectivo observacional y analítico, realizado en pacientes adultos hospitalizados con diagnóstico inicial de cáncer del tracto digestivo. Las variables estudiadas fueron el estado nutricional y la capacidad funcional. Se utilizó estadística descriptiva y asociación con razón de momios (odds ratio [OR]) en paquete estadístico SPSS 14.0. Resultados: Se incluyeron 57 pacientes, de los cuales el 96\% presentaron pérdida de peso. Usando la evaluación global subjetiva (EGS) como método de tamizaje se determinó que el $82.5 \%$ de la población estaba desnutrida, y mediante pruebas bioquímicas e inmunológicas lo estaban el $82 \%$ y el 65\%, respectivamente. La capacidad funcional se evaluó mediante el índice de Karnofsky, encontrando que el $75.5 \%$ de la población tiene alguna limitación en la actividad. Los resultados muestran una asociación entre desnutrición por EGS y limitación en la capacidad funcional $\left(\chi^{2}=1.56 ; p=0.212 ;\right.$ OR: 2.46; intervalo de confianza del 95\% [IC 95\%]: 0.581-10.465). Además, se observó una asociación entre el recuento total de linfocitos y la capacidad funcional $\left(\chi^{2}=6.94 ; p=0.008 ;\right.$ OR: 5.23; IC 95\%: 1.441-19.025). Conclusiones: La desnutrición en pacientes con cáncer de tracto digestivo se asocia con limitación en la capacidad funcional.

PALABRAS CLAVE: Evaluación global subjetiva. Desnutrición. Índice de Karnofsky. Capacidad funcional. Cáncer.

\begin{abstract}
Objective: To determine the nutritional status and its association with functional capacity in patients with digestive tract cancer. Methods: We retrospectively studied all adult patients hospitalized who were diagnosed as having a cancer of the digestive tract. Nutritional status and functional capacity were assessed. Descriptive statistic and odds ratio were used to determine the association in SPSS 14.0. Results: 57 patients were included, 96\% had weight loss. Using subjective global assessment (SGA) as a method of screening, $82.5 \%$ of the patients were found malnutrition and by biochemical and immunological test $82 \%$ and $65 \%$ respectively. Functional capacity was assessed by Karnofsky index, finding that $75.5 \%$ of the patients have some activity limitation. Results show an association between malnutrition by SGA and limitation in functional capacity $\left(\chi^{2}=1.56 ; p=0.212\right.$; OR: 2.46; 95\% confidence interval [95\% Cl]: 0.581-10.465). In addition, we observe an association between the total lymphocyte count and limitation in functional capacity $\left(\chi^{2}=6.94 ; p=0.008\right.$; OR: 5.23; 95\% Cl: 1.441-19.025). Conclusions: Malnutrition in patients with digestive tract cancer was associated with limitation in functional capacity.
\end{abstract}

KEY WORDS: Subjective global assessment. Malnutrition. Karnofsky index. Functional capacity. Cancer.

\footnotetext{
Correspondencia:

Elizabeth Pérez Cruz

Av. Instituto Politécnico Nacional, 5160

Magdalena de las Salinas

C.P. 07760, Ciudad de México, México

E-mail: pece_liz@hotmail.com
}

Fecha de recepción: 18-10-2016

Fecha de aceptación: 01-12-2016

DOI:10.24875/GMM.17002776
Gac Med Mex. 2017;153:575-580

Contents available at PubMed www.gacetamedicademexico.com 


\section{Introducción}

El cáncer es una enfermedad crónica, incapacitante y de gran mortalidad. La Organización Mundial de la Salud (OMS) estima que, para el año 2030, alrededor de 12 millones de personas morirán a causa de este padecimiento ${ }^{1}$, destacando los tumores del tracto digestivo². En México, el cambio en los hábitos alimentarios ha ido de la mano con el incremento de estas neoplasias, entre las que el cáncer gástrico ocupa el primer lugar, seguido por el cáncer de colon y recto ${ }^{3}$.

La desnutrición en el paciente con cáncer resulta de múltiples factores a menudo relacionados con la anorexia, la caquexia y la sensación de saciedad temprana que suelen padecer las personas con cáncer. Ambos están íntimamente relacionados, de modo que la enfermedad puede causar desnutrición y esta, a su vez, puede influir de manera negativa en la enfermedad ${ }^{4}$. Si la desnutrición no es tratada oportunamente, puede conllevar emaciación creciente, debilidad, reducción de la síntesis de proteínas y pérdida de la masa muscular, con lo que afecta directamente la calidad de vida del paciente oncológico e incrementa la mortalidad ${ }^{5-7}$.

Existen diversas herramientas diseñadas para el tamizaje y la evaluación nutricional. La Evaluación Global Subjetiva (EGS), desarrollada en los años 1980 en el Hospital General de Toronto, ha mostrado ser una herramienta altamente confiable y fácilmente reproducible, con una sensibilidad y una especificidad mayores incluso que parámetros tradicionales como los valores de albúmina y de transferrina para evaluar el estado nutriciona|8,9. El índice de Karnofsky (IK) se utilizó por primera vez en la década de 1950 para valorar el estado funcional en pacientes oncológicos sometidos a quimioterapia. Desde entonces ha sido utilizado en diversos estudios clínicos para valorar el estado funcional, como predictor de evolución, supervivencia e incluso como indicador de calidad de vida $^{10-12}$.

En pacientes con cáncer se ha relacionado la puntuación del IK con el índice de Quetelet (IQ), observando un incremento en la sobrevida cuanto más alto se encuentre este último ${ }^{13}$. Algunos estudios muestran asociación entre la desnutrición y la ocurrencia de complicaciones asociadas a los cambios en la capacidad funcional. También se ha descrito la pérdida de peso como un indicador de pronóstico precario en estos pacientes, relacionándose con disminución de la capacidad funcional, lo que nos sugiere una posible relación directa con la presencia de desnutricón $^{2,3}$. El objetivo de este estudio fue conocer el estado nutricional y su asociación con la capacidad funcional en pacientes con cáncer del tracto digestivo.

\section{Métodos}

Estudio retrospectivo observacional y analítico, realizado en pacientes adultos que ingresaron al área de hospitalización de oncología de un hospital federal de referencia de la Ciudad de México durante los años 2011 y 2012. Se incluyeron pacientes de ambos sexos con diagnóstico inicial de cáncer del tracto digestivo; es decir, menos de un año desde el inicio de los síntomas al diagnóstico y sin tratamiento oncológico previo. Se excluyeron aquellos pacientes con datos incompletos.

Las variables estudiadas fueron el estado nutricional y la capacidad funcional, evaluados al ingreso del paciente. También se determinó antropometría, albúmina y recuento total de linfocitos. Las variables demográficas fueron la edad, el sexo, la escolaridad, el tabaquismo, el alcoholismo y la comorbilidad.

El estado nutricional se determinó mediante la EGS, la cual se obtuvo de los registros de la unidad de soporte nutricional, que de manera sistemática se hacen a todos los pacientes dentro de las 24-72 horas posteriores a su admisión. Este método combina aspectos de la historia clínica (pérdida de peso, cambios en la ingesta alimentaria, síntomas gastrointestinales y cambios en la capacidad funcional) y de la exploración física (pérdida de masa grasa y muscular, presencia de edema sacro o ascitis). Los pacientes se clasificaron en tres situaciones nutricionales distintas: bien nutridos, desnutrición moderada y desnutrición grave $^{14}$.

La capacidad funcional se midió mediante el IK. Los puntajes de la escala oscilan entre 0 y 100 , donde un puntaje alto significa que el paciente tiene mejor capacidad para realizar actividades cotidianas (10: paciente moribundo; 100: paciente capaz de llevar una vida normal, independiente y sin asistencia). Los pacientes se clasificaron en dos grupos: sin limitación funcional (IK $\geq 80)$ y con limitación funcional $(\mathrm{IK} \leq 70)^{10,11}$.

Las mediciones antropométricas registradas incluyeron talla $(\mathrm{cm})$ y peso $(\mathrm{kg}), \mathrm{IQ}\left(\mathrm{kg} / \mathrm{m}^{2}\right)$ y pérdida de peso en los últimos 6 meses, la cual se calculó mediante la expresión: 
Pérdida de peso $(\%)=\frac{(\text { peso actual-peso habitual }) \times 100}{\text { peso habitual }}$

Los parámetros bioquímicos e inmunológicos nutricionales complementaron la evaluación nutricional y se obtuvieron del expediente clínico. Considerando los valores de albúmina, los pacientes se clasificaron como: sin desnutrición (> $3.5 \mathrm{~g} / \mathrm{dl})$ ), con desnutrición leve (3.5-2.8 $\mathrm{g} / \mathrm{dl})$, con desnutrición moderada $(2.1-2.7 \mathrm{~g} / \mathrm{dl})$ y con desnutrición grave $(<2.1 \mathrm{~g} / \mathrm{dl})$. Los puntos de corte considerados para la clasificación según el recuento total de linfocitos [(\%linfocitos $x$ leucocitos)/100] fueron $>1500 \mathrm{~mm}^{3}$ para normal, 1200-1500 mm³ para desnutrición leve, $800-1199 \mathrm{~mm}^{3}$ para desnutrición moderada $\mathrm{y}<800 \mathrm{~mm}^{3}$ para desnutrición grave.

El análisis de los datos se realizó con el paquete estadístico SPSS versión 14. 0 (SPSS Inc. Chicago, IL. USA). Se utilizó estadística descriptiva. Las variables cualitativas se analizaron mediante frecuencias y porcentajes; las variables cuantitativas, mediante media y desviación estándar. La prueba utilizada para analizar la asociación entre el estado nutricional y la funcionalidad fue con razón de momios y ji al cuadrado. El nivel de significancia que se adoptó para las comparaciones fue $\alpha=5 \%$. El estudio fue aprobado por el Comité de Ética e Investigación con registro institucional HJM2013/11R.

\section{Resultados}

Se evaluaron finalmente 57 pacientes con diagnóstico reciente de cáncer del tracto digestivo. El promedio de edad fue de $57.8 \pm 14.5$ años, siendo el $54 \%$ hombres y el $46 \%$ mujeres. El $96 \%$ de los pacientes refirieron pérdida de peso, la cual se estimó en un promedio de $13.18 \pm 8.73 \%$. El $35 \%$ de la población tuvo una pérdida del $11-20 \%$ del peso habitual, el $17 \%$ de la población refirió una pérdida de peso del
$20-30 \%$, y el $34 \%$ tuvo una pérdida de peso del $1-10 \%$; solo el $4 \%$ de la población estudiada no registró pérdida de peso. Las características demográficas se muestran en la tabla 1.

Los tipos de cáncer que predominaron fueron los de colon (35\%), gástrico (23\%), páncreas $(9 \%)$, esófago $(7 \%)$, intestino delgado (2\%) y otros (24\%). El $94.8 \%$ de los pacientes se encontraban en estadio clínico II y III; solo el $1.7 \%$ en estadio clínico I y el $3.5 \%$ en estadio clínico II.

La desnutrición afectó al $82.5 \%$ de la población medida por EGS, de los cuales el $21 \%$ presentaron desnutrición moderada y el $61.5 \%$ desnutrición grave. Por parámetros bioquímicos, como la albúmina, el $18 \%$ se clasificó dentro de lo normal, el $24 \%$ con depleción leve, el $21 \%$ moderada y el $37 \%$ grave, con un promedio de $3.2 \pm 0.79 \mathrm{~g} / \mathrm{dl}$. Considerando parámetros inmunológicos, como el recuento total de linfocitos, el $65 \%$ presentó desnutrición, el 14\% desnutrición leve, el $21 \%$ desnutrición moderada y el $30 \%$ desnutrición grave.

En relación con la capacidad funcional determinada por el IK, el $24.5 \%$ de la población se encontraba realizando sus actividades prácticamente de manera normal, en tanto que el $75.5 \%$ presentó alguna limitación en la actividad. Al realizar el subanálisis como indicador global de autosuficiencia, de ese $75.5 \%$ encontramos un 35\% con alguna limitación en puntuaciones de 80; un 35\% con actividad limitada, es decir, puntuaciones entre 50 y 70 ; y un $5.5 \%$ con incapacidad de autocuidado y puntuaciones entre $10 \mathrm{y}$ 40.

La asociación entre el estado nutricional por EGS y la capacidad funcional de la población se muestra en la tabla 2. De los pacientes que mostraron desnutrición, el $78.7 \%$ tenían limitaciones en la actividad $\left(\chi^{2}=1.56 ; p=0.212\right)$. En la tabla 3 se muestra la asociación entre el estado nutricional, clasificado por el recuento total de linfocitos, y la capacidad

Tabla 1. Caracterización demográfica de la población estudiada

\begin{tabular}{lccc}
\hline Característica & Global $\mathbf{n}=\mathbf{5 7}$ & Sin desnutrición $\mathbf{n}=\mathbf{1 0}$ & Con desnutrición $\mathbf{n}=\mathbf{4 7}$ \\
\hline Edad (años) & $57.8 \pm 14.5$ & $54.9 \pm 5.71$ & $58.43 \pm 14.4$ \\
Sexo (H/M) & $31 / 26$ & $4 / 6$ & $27 / 20$ \\
Pérdida de peso (\%) & $13.18 \pm 8.73$ & $5.9 \pm 6.9$ & $14.7 \pm 8.33$ \\
IQ $\left(\mathrm{kg} / \mathrm{m}^{2}\right)$ & $22.6 \pm 3.8$ & $21.3 \pm 3.4$ & $22.8 \pm 3.8$ \\
Tabaquismo presente & $27(47.4 \%)$ & $7(70 \%)$ & $20(42.5 \%)$ \\
\hline
\end{tabular}


funcional. De los que mostraron desnutrición, el 56.1\% tenían limitaciones en la actividad $\left(\chi^{2}=6.94 ; p=0.008\right)$.

La tabla 4 muestra las relaciones entre la capacidad funcional y las variables predictoras del estudio.

\section{Discusión}

La desnutrición en los pacientes con cáncer es una realidad, y la pérdida de peso ha sido un indicador directo de esta. Algunos estudios han mostrado que, al momento del diagnóstico, el $80 \%$ de los pacientes con cáncer gastrointestinal ya han experimentado una pérdida de peso significativa, en general de al menos el $10 \%$ del peso corporal, en un periodo de 6 me$\operatorname{ses}^{4,5,15}$. En nuestra población de estudio reportamos un porcentaje mayor de pacientes con pérdida de peso y con pérdidas promedio superiores al $10 \%$ en 6 meses. La mayor parte de nuestros pacientes presentaron cáncer de colon, seguido del cáncer

Tabla 2. Asociación entre el estado nutricional por EGS y la capacidad funcional según el IK

\begin{tabular}{|c|c|c|c|}
\hline \multirow{2}{*}{$\begin{array}{l}\text { Capacidad } \\
\text { funcional }\end{array}$} & \multicolumn{2}{|c|}{ Estado nutricional } & \multirow[t]{2}{*}{ Total } \\
\hline & Sin desnutrición & Con desnutrición & \\
\hline No limitada & 4 & 10 & 14 \\
\hline Limitada & 6 & 37 & 43 \\
\hline Total & 10 & 47 & 57 \\
\hline
\end{tabular}

Tabla 3. Asociación entre el estado nutricional por pruebas inmunológicas (recuento total de linfocitos) y la capacidad funcional según el IK

\begin{tabular}{|c|c|c|c|}
\hline \multirow{2}{*}{$\begin{array}{l}\text { Capacidad } \\
\text { funcional }\end{array}$} & \multicolumn{2}{|c|}{ Estado nutricional } & \multirow[t]{2}{*}{ Total } \\
\hline & Sin desnutrición & Con desnutrición & \\
\hline No limitada & 9 & 5 & 14 \\
\hline Limitada & 11 & 32 & 43 \\
\hline Total & 20 & 37 & 57 \\
\hline
\end{tabular}

Tabla 4. Relaciones entre la capacidad funcional y variables bioquímicas y clínicas

\begin{tabular}{lcc}
\hline Característica & $\mathbf{r}$ & $\mathbf{p}$ \\
\hline Colesterol & 0.174 & 0.490 \\
Albúmina & 0.400 & 0.826 \\
Linfocitos & 0.255 & 0.055 \\
Estadio del cáncer & 0.489 & 0.001 \\
\hline
\end{tabular}

gástrico, lo cual puede justificar esta mayor pérdida de peso. Lo anterior, reforzado por los hallazgos de Moon, et al. ${ }^{16}$, aunado a la reducción de la ingesta, la disfagia y la mucositis que incluso se ven acentuados durante los tratamientos oncológicos, principalmente la quimioterapia ${ }^{17}$.

Al evaluar a los pacientes mediante el IQ observamos que aunque más de la mitad presentaban un índice normal o incluso mayor, al aplicar la EGS vemos que más de dos terceras partes de la población presentaban algún grado de desnutrición. Tolentino, et al. ${ }^{18}$ reportaron, en su estudio en pacientes con cáncer, que aunque la pérdida de peso es bastante común, el IQ disminuyó principalmente en presencia de tumores gastrointestinales en comparación con tumores no gastrointestinales. En nuestro estudio determinamos que, aunque el IQ era similar en ambos grupos, la pérdida de peso sí fue mayor en el grupo con desnutrición determinada por EGS. Por lo tanto, el IQ no es un indicador sensible para detectar desnutrición. La utilización de la EGS sigue siendo un método práctico que nos permite distinguir entre pacientes bien nutridos y con desnutrición, sin necesidad de recurrir a medidas antropométricas o de laboratorio.

La desnutrición como deficiencia de múltiples nutrimentos altera las funciones en las que se encuentra implicado el sistema inmunitario. Para este fin, la determinación del número de leucocitos totales y de los diferentes tipos de células blancas es un procedimiento sencillo que permite obtener información útil y que se ha asociado con el pronóstico del cáncer ${ }^{19-21}$. Incluso los cocientes neutrófilo/linfocito y plaquetas/ linfocitos se han estudiado como predictores de agresividad, como de mal pronóstico, debido a que los neutrófilos son fuente del factor de crecimiento endotelial, el cual participa en la angiogénesis y aumenta la capacidad de diseminación del tumor. Además, la elevación de los marcadores inflamatorios sistémicos se asocia a linfocitopenia y a una respuesta alterada de los linfocitos T hacia el tumor ${ }^{22-24}$.

Considerando que las funciones de las células del sistema inmunitario están influenciadas por sustancias antioxidantes y prooxidantes, y que las células cancerosas sufren perturbaciones en el balance energético de electrones, determinamos los valores totales de linfocitos de los pacientes como marcador de desnutrición y observamos que más de la mitad presentaban algún grado desnutrición, además de encontrar una clara asociación con la capacidad funcional, situación que no constatamos en otros indicadores bioquímicos como la albúmina o el colesterol. 
En el paciente con cáncer resultan preocupantes las alteraciones del estado nutricional, especialmente la desnutrición, debido a que diversos estudios la han relacionado con la tolerancia a los tratamientos, la calidad de vida y la sobrevida. Para valorar la repercusión del estado nutricional y su posible asociación con la capacidad funcional utilizamos el IK. Si bien existen diversas escalas para medir la capacidad funcional, el IK ha sido catalogado como el mejor método por su poder predictor de supervivencia, permitiendo determinar el nivel de actividad y dependencia de los pacientes en relación con sus cuidados médicos ${ }^{25,26}$. La Escala Funcional ECOG (Eastern Cooperative Oncology Group)/OMS, aplicada por primera vez en 1960 a pacientes que recibieron quimioterapia, ha mostrado una alta correlación con el IK. Los valores del coeficiente de correlación de Spearman obtenidos fueron de 0.85 $(p>0.0001)$ en el estudio de Loperinzi, et al. ${ }^{26}$, y de 0.87 ( $p>0.0001$ ) en el estudio de Buccheri, et al. ${ }^{27}$. La Escala Funcional Paliativa es una escala de medición de la capacidad funcional diseñada específicamente para pacientes con cuidados paliativos, al igual que la Escala de Evaluación Funcional de Edmonton. La Escala Funcional Paliativa es una modificación del IK y por lo tanto cuenta con una alta correlación (coeficiente de Spearman de 0.94). En nuestro estudio observamos que, aunque los valores del IK no son tan bajos como los reportados en otras series, los pacientes presentan ya un grado de limitación en la actividad y cierta dependencia; así, solo el 5.5\% presentó un $\mathrm{IK}<50$, con una supervivencia esperada inferior a 6 meses $^{28,29}$. Observamos, además, una asociación entre el estado nutricional por EGS y la capacidad funcional por IK. Este hecho, debido a que la desnutrición ligada a la enfermedad, al estadio e incluso a los tratamientos que recibe el paciente, produce disminución en la masa grasa y disminución de la fuerza muscular, con efecto inmediato en la reducción de la actividad física y la capacidad funcional de estos pacientes. Ottery ${ }^{30}$, en un estudio de pacientes con cáncer gastrointestinal, identificó que el estado nutricional, el desarrollo de la enfermedad y la ubicación del tumor eran factores independientes sobre la funcionalidad y la calidad de vida.

Las escalas para valorar la capacidad funcional han mostrado su poder predictivo de supervivencia, el cual puede atribuirse a las estimaciones clínicas. Aunque Maltoni, et al. ${ }^{31}$ han puesto en entredicho esta aseveración, en nuestro estudio observamos una correlación negativa entre la capacidad funcional y el estadio clínico del cáncer, de tal manera que depende de la evolución del paciente, y cuanto más avanzada está la enfermedad, mejor es la estimación pronóstica realizada con la medición de la capacidad funcional.

La identificación del estado nutricional basal de un paciente con cáncer y la intervención nutricional influyen en el éxito del tratamiento antineoplásico y en su pronóstico general. Aunque el soporte nutricional contribuye a normalizar el estado nutricional, mejorar la funcionalidad y la calidad de vida, no debe olvidarse el enfoque terapéutico de aquellos pacientes que se encuentren en estadios tempranos y localmente avanzados con potencial curativo; en tanto, aquellos en estadios avanzados o metastásicos serán de control de síntomas, mejora de la calidad de vida y aumento en la supervivencia global ${ }^{32-34}$.

Finalmente, es necesario que en nuestros centros contemos con herramientas de valoración nutricional que nos permitan la identificación oportuna de los pacientes en riesgo de desnutrición o con desnutrición; así mismo, de una escala de valoración del estado funcional y de calidad de vida, ya que resulta indispensable desde el punto de vista terapéutico de acuerdo con los objetivos del mismo.

\section{Conclusiones}

La prevalencia de desnutrición en nuestro medio es alta en los pacientes con cáncer de tracto digestivo. El estado nutricional determinado por la EGS y por recuento total de linfocitos guarda asociación con la capacidad funcional.

\section{Conflictos de intereses}

No existe ningún conflicto de intereses. No existe ninguna relación económica, personal, política o académica, ni se han recibido beneficios de dinero, bienes, hospitalidad o subsidios de ninguna fuente que pudieran influir en la presentación de los resultados de este estudio.

\section{Bibliografía}

1. American Cancer Society. Cancer facts and figures 2011. Atlanta: American Cancer Society; 2011. p. 1-55.

2. McMahon K, Decker G, Ottery FD. Integrating proactive nutritional assessment in clinical practices to prevent complications and cost. Semin Oncol. 1998;25(2 Suppl 6):20-7.

3. Abdo Francis JM. Cáncer del aparato digestivo. Revista Médica Hospital General de México. 2010;73:7.

4. Bruera $E$. ABC of palliative care. Anorexia, cachexia, and nutrition. BMJ. 1997;315:1219-22.

5. Van Cutsem E, Arends J. The causes and consequences of cancer-associated malnutrition. Eur J Oncol Nurs. 2005;9(Suppl 2):S51-63.

6. McMillan DC. An inflammation-based prognostic score and its role in the nutrition-based management of patients with cancer. Proc Nutr Soc. 2008;67:257-62. 
7. Eldridge B, Rock CL, McCallum PD. Nutrition and the Patient with Cancer. In: Nutrition in the Prevention and Treatment of Disease. Ed. Coulston AM Rock CL, Monsen ER. Academic Press, San Diego, 2001. pg. 397-412.

8. Ek AC, Unosson M, Larsson J, et al. Interrater variability and validity in subjective nutritional assessment of elderly patients. Scand J Caring Sci. 1996;10:163-8

9. Enia G, Sicuso C, Alati G, et al. Subjective Global Assessment of nutrition in dialysis patients. Nephrol Dial Transplant.1993;8:1094-8.

10. Karnofsky DA, Abelman WH, Craver LF, et al. The use of nitrogen mustards in the palliative treatment of cancer. Cancer. 1948;1:634-56.

11. Karnofsky DA, Burchenal JH. The clinical evaluation of chemotherapeutic agents in cancer. En: MacLeod CM, editor. Evaluation of chemotherapeutic agents. New York: Columbia University Press; 1949. p. 191-205

12. Puiggròs $\mathrm{C}$, Lecha $\mathrm{M}$, Rodríguez $\mathrm{T}$, et al. Karnofsky Index as a mortality predicting factor in patients on home-based enteral nutrition. Nutr Hosp. 2009;24:156-60.

13. Ruiz-Delgado GJ, Lutz-Presno JA, Alarcón-Urdaneta C, et al. Body mass index as an indicator of prognosis in patients undergoing allogenic hematopoietic stem cell transplantation. Rev Hematol Mex. 2011:12:28-31.

14. Detsky AS, McLaughlin JR, Baker JP, et al. What is subjective global assessment of nutritional status? JPEN J Parenter Enteral Nutr. 1987;11:8-13.

15. Rivadeneira DE, Evoy D, Fahey TJ 3rd, et al. Nutritional support of the cancer patient. CA Cancer J Clin. 1998;48:69-80.

16. Moon $\mathrm{H}$, Roh JL, Lee SW, et al. Prognostic value of nutritional and hematologic markers in head and neck squamous cell carcinoma treated by chemoradiotherapy. Radiother Oncol. 2016;118:330-4.

17. Cho YW, Roh JL, Jung JH, et al. Prediction of prosttreatment significant body weight loss and its correlation with disease free survival in patients with oral squamous cell carcinomas. Nutr Cancer. 2013;65:417-23.

18. Tolentino R, Quizon O, Llido L. Nutritional status of patients with malignancy of the gastrointestinal tract and other malignancies - comparison of characteristics and pattern: a two-year study in a private tertiary care hospital in the Philippines. Philip J Oncology. 2007;8:37-44.

19. Huang SH, Waldron JN, Milosevic M, et al. Prognostic value of pretreatment circulating neutrophils, monocytes, and lymphocytes in oropharyngeal cancer stratified by human papillomavirus status. Cancer. 2015;121:545-55.

20. De Carvalho TM, Miguel Marin D, da Silva CA, et al. Evaluation of patients with head and neck cancer performing standard treatment in relation to body composition, resting metabolic rate, and inflammatory cytokines. Head Neck. 2015;37:97-102.
21. Rassouli A, Saliba J, Castano R, et al. Systemic inflammatory markers as independent prognosticators of head and neck squamous cell carcinoma. Head Neck. 2015;37:103-10.

22. Cannon NA, Meyes J, lyengar P, et al. Neutrophil-lymphocyte and platelet-lymphocyte ratios as prognostic factors after stereotactic radiation therapy for early-stage non-small-cell lung cancer. J Thorac Oncol. 2015;10:280-5.

23. Fan $W$, Zhang $Y$, Wang $Y$, et al. Neutrophil-to-lymphocyte and platelet-to-lymphocyte ratios as predictors of survival and metastasis for recurrent hepatocellular carcinoma after transarterial chemoembolization. PLos One. 2015;10:e0119312.

24. Kim EY, Lee JW, Yoo HMY. The platelet-to-lymphocyte ratio versus neutrophil-to-lymphocyte ratio: which is better as a prognostic factor in gastric cancer? Ann Surg Oncol. 2015;22:4363-70.

25. Mor V, Laliberte L, Morris JN, et al. The Karnofsky Performance Status Scale: an examination of its reliability and validity in a research setting. Cancer. 1984;53:2002-7.

26. Loperinzi CL, Laurie JA, Wieand HS, et al. Prospective evaluation of prognostic variables from patient-completed questionnaires. J Clin Oncol. 1994;12:601-7.

27. Buccheri G, Ferrigno D, Tamburini M. Karnofsky and ECOG performance status scoring in lung cancer: a prospective, longitudinal study of 536 patients from a single institution. Eur $\mathrm{J}$ Cancer. 1996;32A:1531-41

28. Yates JW, Chalmer B, McKegney FP. Evaluation of patients with advanced cancer using the Karnofsky Performance Status. Cancer. 1980; 45:2220-4.

29. Maltoni M, Pirovano M, Scarpi E, et al. Prediction of survival of patients terminally ill with cancer. Results of an Italian prospective multicentric study. Cancer. 1995;75:2613-22.

30. Ottery FD. Supportive nutrition to prevent cachexia and improve quality of life. Semin Oncol. 1995;22(Suppl 3):98-111.

31. Maltoni M, Nanni O, Derni S, et al. Clinical prediction of survival is more accurate than the Karnofsky performance status in estimating life span of the terminally ill cancer patients. Eur J Cancer. 1994;30:764-6.

32. McMillan DC. An inflammation-based prognostic score and its role in the nutrition-based management of patients with cancer. Proc Nutr Soc. 2008;67:257-62.

33. Gârtner S, Krûger J, Aghdassi AA, et al. Nutrition in pancreatic cancer: a review. Gastrointest Tumors. 2015;2:195-202.

34. Rosania R, Chiapponi C, Malfertheiner $P$, et al. Nutrition in patients with gastric cancer: an update. Gastrointest Tumors. 2015;2:178-87. 